1. George AL Jr. Inherited disorders of voltage-gated sodium channels. JClin Invest. 2005;115(8):1990-1999.

2. Jurkat-Rott K, Lehmann-Horn F. Muscle channelopathies and critical points in functional and genetic studies. J Clin Invest. 2005;115(8):2000-2009.

3. Meisler MH, Kearney JA. Sodium channel mutations in epilepsy and other neurological disorders. J Clin Invest. 2005;115(8):2010-2017.

4. Moss AJ, Kass RS. Long QT syndrome: from channels to cardiac arrhythmias. J Clin Invest. 2005 115(8):2018-2024

5. Jentsch TJ, Maritzen T, Zdebik AA. Chloride channel diseases resulting from impaired transepithelial transport or vesicular function. J Clin Invest. 2005;115(8):2039-2046.

6. Cannon SC, George AL. Pathophysiology of myotonia and periodic paralysis. In: Asbury AK, Mckhann GM, McDonald WI, Goadsby PJ, McArthur JC, eds. Diseases of the Nervous System, Clinical Neuroscience and Therapentic Principles. 3rd ed. Cambridge, United Kingdom: Cambridge University Press; 2002:1183-1206.

7. Rüdel R, Lehmann-Horn F, Ricker K, Kuther G Hypokalemic periodic paralysis: in vitro investigation of muscle fiber membrane parameters. Muscle Nerve. 1984;7(2):110-120.

8. Lehmann-Horn F, et al. Two cases of adynamia epi- sodica hereditaria: In vitro investigation of muscle cell membrane and contraction parameters. Muscle Nerve. 1983;6(2):113-121

9. Armstrong CM, Hille B. Voltage-gated ion channels and electrical excitability. Neuron. 1998; 20(3):371-380.

10. Swartz KJ. Sensing voltage across lipid membranes. Nature. 2008;456(7224):891-897.

11. Bezanilla F. How membrane proteins sense voltage. Nature Rev Mol Cell Biol. 2008;9(4):323-332.

12. Yang NB, George AL Jr, Horn R. Molecular basis of charge movement in voltage-gated sodium channels. Neuron. 1996;16(1):113-122.

13. Larsson HP, Baker OS, Dhillon DS, Isacoff EY. Transmembrane movement of the shaker $\mathrm{K}^{+}$channel S4. Neuron. 1996;16(2):387-397.

14. Starace DM, Stefani E, Bezanilla F. Voltage-dependent proton transport by the voltage sensor of the shaker $\mathrm{K}^{+}$channel. Neuron. 1997;19(6):1319-1327.

15. Sokolov S, Scheuer T, Catterall WA. Gating pore current in an inherited ion channelopathy. Nature. 2007;446(7131):76-78.

16. Sokolov S, Scheuer T, Catterall WA. Depolarization-activated gating pore current conducted by mutant sodium channels in potassium-sensitive normokalemic periodic paralysis. Proc Natl Acad Sci
US A. 2008;105(50):19980-19985.

17. Struyk AF, Cannon SC. A Na ${ }^{+}$channel mutation linked to hypokalemic periodic paralysis exposes a proton-selective gating pore. J Gen Physiol. 2007; 130(1):11-20.

18. Struyk AF, Markin VS, Francis D, Cannon SC. Gating pore currents in DIIS4 mutations of $\mathrm{Na}_{\mathrm{V}} 1.4$ associated with periodic paralysis: saturation of ion flux and implications for disease pathogenesis. J Gen Physiol. 2008;132(4):447-464.

19. Wu F, et al. A sodium channel knockin mutant (Nav1.4-R669H) mouse model of hypokalemic periodic paralysis. J Clin Invest. 2011;121(10):4082-4094.

20. Wu F, et al. A calcium channel mutant mouse model of hypokalemic periodic paralysis. $J$ Clin Invest. 2012;122(12):4580-4591.

21. Jurkat-Rott K, Groome J, Lehmann-Horn F. Pathophysiological role of omega pore current in channelopathies. Front Pharmacol. 2012;3:112.

22. Gosselin-Badaroudine P, et al. A Proton Leak current through the cardiac sodium channel is linked to mixed arrhythmia and the dilated cardiomyopathy phenotype. PLoS One. 2012;7(5):e38331.

23. Ahern CA, Horn R. Specificity of charge-carrying residues in the voltage sensor of potassium channels. J Gen Physiol. 2004;123(3):205-216.

\title{
aKlotho: FGF23 coreceptor and FGF23-regulating hormone
}

\author{
Harald Jüppner ${ }^{1}$ and Myles Wolf ${ }^{2}$
}

${ }^{1}$ Endocrine Unit and Pediatric Nephrology Unit, Massachusetts General Hospital, Boston, Massachusetts, USA. ²Division of Nephrology and Hypertension, Department of Medicine, University of Miami Miller School of Medicine, Miami, Florida, USA.

\begin{abstract}
Low levels of phosphate can disrupt bone ossification and predispose to fractures. FGF23 is one of the major determinants of phosphate homeostasis, acting to increase urinary phosphate excretion. However, the regulation of FGF23 is incompletely understood. In this issue of the JCI, Smith et al. show that the cleaved form of $\alpha$ Klotho, the membrane-bound form of which is an FGF23 coreceptor, serves as a novel endocrine regulator of phosphate homeostasis, capable of inducing FGF23 production in osteocytes.
\end{abstract}

Phosphate is a critical component of bone, and it serves numerous biological functions in the synthesis of DNA and membrane lipids, protein modifications, energy metabolism, and second messenger formation. FGF23 and parathyroid hormone (PTH) are the major regulators of phosphate homeostasis $(1,2)$. Both hormones increase urinary phosphate excretion by reducing proximal tubular expression of the sodi-

Conflict of interest: Myles Wolf has received research support or honoraria from Abbott Laboratories, Amgen, DiaSorin, Kai Pharmaceuticals, Luitpold Pharmaceuticals Inc., Mitsubishi, Sanofi, and Shire. Harald Jüppner has received honoraria from Kai Pharmaceuticals, Roche, Kyowa Hakko Kirin, Genzyme, Amgen, and Pfizer; he is a coinventor of a patent describing a technique to measure FGF23.

Citation for this article: J Clin Invest. 2012; 122(12):4336-4339. doi:10.1172/JCI67055. um-dependent phosphate cotransporters, NPT2a and NPT2c, thereby lowering serum phosphate levels. However, in contrast to FGF23, which decreases serum levels of 1,25-dihydroxyvitamin D (1,25D) by inhibiting renal $1 \alpha$-hydroxylase and stimulating 24-hydroxylase, PTH increases renal production of $1,25 \mathrm{D}$, which consequently enhances the absorption of phosphate (and calcium) from the intestinal tract (3).

FGF23 belongs to the subfamily of endocrine FGFs and mediates its phosphateregulating actions in the kidney through FGF receptors (FGFRs), most prominently FGFR1. These actions require the transmembrane form of $\alpha$ Klotho ( $\mathrm{mKL}$ ), which acts as the coreceptor that enhances the binding affinity of FGF23 to different FGFRs $(4,5)$. Ablation of $\alpha$ Klotho in mice leads to severe hyperphosphatemia, elevated levels of $1,25 \mathrm{D}$, hypercalcemia, diffuse soft tissue calcifications, accelerated aging, and premature death (6). These findings are largely indistinguishable from those observed in FGF23 loss-of-function models $(7,8)$.

Osteocytes are the primary cellular source of FGF23 after the fetal period (9), but little is known about their regulation and secretion. Although discovery of the molecular causes of monogenic hypophosphatemic rickets disorders has helped to identify a set of bone-derived proteins that decrease FGF23 production, the mechanisms of how these inhibitors suppress FGF23 remain largely unknown. Furthermore, despite our knowledge that changes in dietary phosphate intake can alter FGF23 levels in healthy individuals $(10,11)$, how serum phosphate or phosphate balance is sensed and how this signal is conveyed to the osteocyte to alter FGF23 expression remains completely unknown.

A novel regulator of FGF23 synthesis In this issue of the JCI, Smith et al. provide intriguing new findings about the 


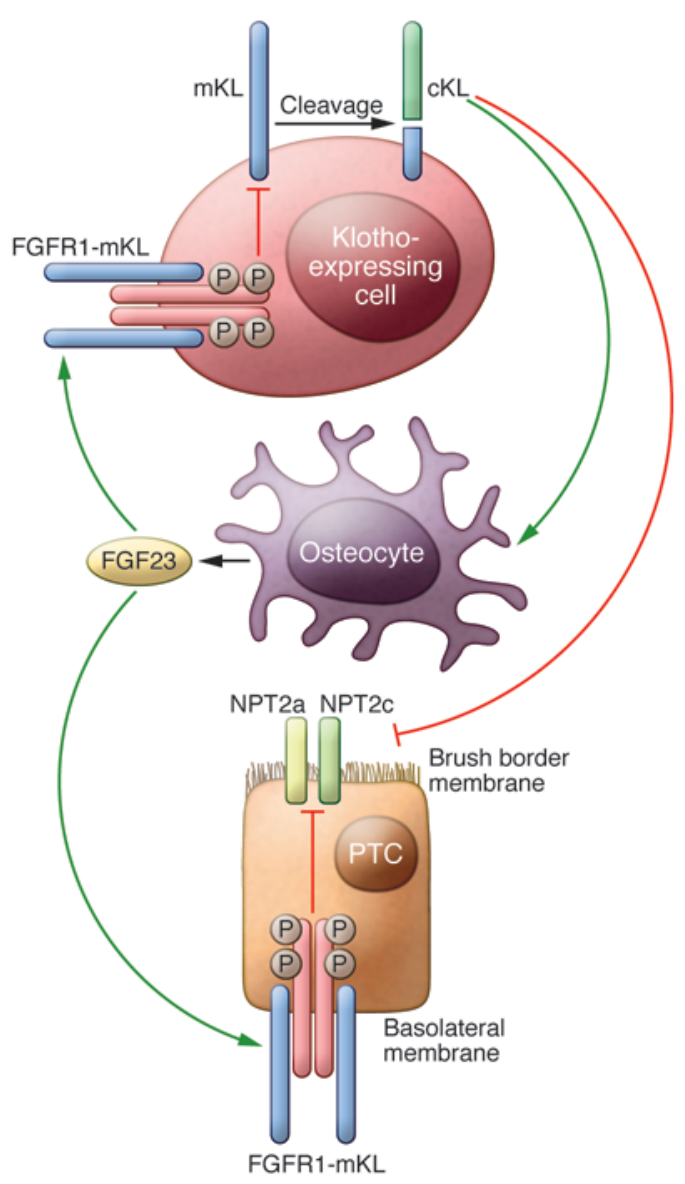

regulation of FGF23 production (12). Using an adenoviral vector, they constitutively overexpressed a naturally occurring cleaved form of $\alpha$ Klotho (cKL; mouse amino acid residues 35-983) that lacks the membrane-spanning domain of fulllength klotho but is distinct from the smaller soluble splice variant (sKL). Mice treated with cKL-expressing virus developed profound hypophosphatemia, associated with a dramatic increase in secretion of intact, biologically active FGF23, resulting in osteomalacia, expanded growth plates, reduced bone mineral density, and a propensity for bone fractures. It is important to emphasize that the magnitude of FGF23 elevation was much higher than that observed in most human diseases and animal models of FGF23-mediated hypophosphatemic syndromes. Indeed, similarly elevated levels are seen only in states of $\alpha$ Klotho deficiency and endstage renal disease in which the marked FGF23 elevation is accompanied by hyperphosphatemia (13). The biochemical profile of mineral metabolism in the animals treated with cKL-expressing virus recapitulated that of a patient in whom a chromosomal translocation resulted in markedly increased circulating cKL levels and FGF23-dependent hypophosphatemic rickets (14).

As observed in the patient with the spontaneously occurring mutation (14), viral cKL expression also led to markedly increased PTH concentrations (12). Although this may have been due to hypocalcemia or FGF23-mediated reduction of $1,25 \mathrm{D}$ driven by reduced Cyp27b1 and increased Cyp24a1 mRNA levels, and thus impaired intestinal calcium absorption, PTH seems to have risen prior to the onset of overt hypocalcemia. Indeed, PTH was significantly elevated 2 weeks after high-dose viral infection, when serum calcium levels were subtly but significantly increased compared with those of control. This suggests that cKL may have direct effects on the parathyroid glands that led to secondary hyperparathyroidism, which is an intriguing hypothesis, since activation of the FGFR1-FGF23-mKL complex in the parathyroid is thought to suppress PTH secretion $(15,16)$.

\section{Figure 1}

Working model of endocrine feedback loops involving FGF23 and Klotho. mKL, which is expressed primarily in distal renal tubules, parathyroids, and choroid plexus, can be cleaved to generate $\mathrm{cKL}$. The resulting hormonal factor increases the osteocytic production of FGF23, which activates the FGFR1$\mathrm{mKL}$ receptor complex in renal proximal tubular cells (PTCs), probably at the basolateral membrane. In addition, $\mathrm{CKL}$ directly deglycosylates NPT2a and NPT2c protein, making it more susceptible to proteases, thereby reducing expression of both transporters in the apical membrane. Not pictured, cKL may serve as a "portable" coreceptor of FGF23, thus enhancing FGF23 signaling in cells such as PTCs, in which $\mathrm{mKL}$ is less abundantly expressed.

\section{A new endocrine negative feedback loop?}

Hu et al. (17) previously showed that injection of recombinant $\mathrm{CKL}$ protein (mouse amino acid residues 31-982) induced hypophosphatemia, an effect that was preserved in Fgf23-null mice. Furthermore, cKL efficiently reduced phosphate uptake in a kidney epithelial cell line in the absence of FGF23. Additional studies led to the conclusion that the direct actions of cKL are most likely related to its glucuronidaselike activity, which deglycosylates NPT2a and probably NPT2c, thus decreasing stability of the transporters in the apical membrane of the proximal renal tubules $(17,18)$. In addition to this phosphaturic activity at the level of the kidney, the findings by Smith et al. now indicate that $\mathrm{cKL}$ is also a novel endocrine regulator of phosphate homeostasis via the induction of FGF23 production (12). This suggests the presence of yet another classic negative endocrine feedback loop in mineral metabolism: FGF23 negatively regulates $\mathrm{mKL}$ expression $(19,20)$, while the cKL increases FGF23 production (Figure 1). 


\section{New questions}

As usual in this rapidly evolving field, the current findings raise a number of new, important questions. For example, it is unclear why hypophosphatemia induced by virally expressed cKL is not self-corrected by secondary reductions of endogenous FGF23 synthesis. This implies that constitutive $\mathrm{cKL}$ expression overrides the effects of inhibitory signals to osteocytes to lower FGF23 levels, or, more provocatively, it could suggest that the serum phosphate level does not actually regulate FGF23 secretion directly. If $\mathrm{cKL}$ is indeed a major regulator of FGF23 production, as suggested by this report, it also remains to be determined why FGF23 levels are so dramatically increased in humans and mice completely lacking $\alpha$ Klotho $(4,21)$. Is FGF23 production in this setting primarily stimulated by the profound elevation in serum phosphate levels or through uninhibited overproduction of $1,25 \mathrm{D}$ ?

One emerging aspect of FGF23 regulation that the study by Smith et al. did not address was FGF23 cleavage within osteocytes, which appears to be a counterregulatory mechanism to maintain normal circulating levels of biologically active FGF23 when mRNA expression is increased. This was initially observed in wild-type mice placed on an iron-deficient diet, which increased Fgf23 mRNA expression in bone, yet led to appearance of FGF23 fragments in bone homogenates and increased circulating levels of C-terminal FGF23 fragments, but did not change intact FGF23 levels (22). Since C-terminal FGF23 levels were neither measured in the study by Smith et al. (12) nor in the patient with increased cKL levels due to the chromosomal translocation (14), we cannot determine whether FGF23 degradation was impaired or whether the mechanism responsible for cleaving intact FGF23 was overwhelmed by the relentless increase in FGF23 expression. The absence of C-terminal FGF23 fragments would thus confirm that cleavage of intact FGF23 is an important mechanism in the regulation of phosphate homeostasis.

Furthermore, it remains to be determined whether and how cKL stimulates FGF23 production. When added together to NIH3T3 cells expressing FGFR1, recombinant cKL and FGF23 triggered a robust increase in ERK1/2 phosphorylation, which is different from earlier findings in HEK293 cells in which FGF23 failed to activate the FGFR1 in the presence of cKL (or sKL) (23).
These disparate findings suggest that $\mathrm{cKL}$ can facilitate FGF23 signaling only in certain cell types, for example, the proximal tubular cells in the kidney. Based on the study by Smith et al., it appears plausible that cKL can act as a hormone that activates FGFR1 signaling in osteocytes, thereby enhancing, directly or indirectly, FGF23 production and consequently urinary phosphate excretion. Alternatively, cKL may activate another distinct, as-of-yet unknown, receptor-signaling system.

\section{Translating the novel observations}

The presented findings raise additional important questions. What is their relevance to normal day-to-day regulation of mineral metabolism, and is it conceivable that more modest changes in circulating levels of cKL than those induced in this extreme model have a prominent role in regulating FGF23 levels in normal human physiology? Does cKL act as a "portable" coreceptor that can bind to FGF23 in the circulation, thereby transforming FGFRs that have low affinity for FGF23 into high-affinity receptors that are capable, at least in some tissues, of efficient signal transduction? Is the reduction in $\mathrm{cKL} \mathrm{lev-}$ els that occurs in chronic kidney disease an adaptive response to reduce markedly elevated FGF23 levels through this new putative endocrine feedback loop? Smith et al. have provided novel insights into the complex regulation of phosphate homeostasis, which will generate new hypotheses regarding the endocrine role of $\mathrm{CKL}$ in the production of FGF23 and its potential therapeutic implications. For example, the presented findings raise the possibility that reducing cKL levels could be a novel therapeutic approach to ameliorate FGF23dependent hypophosphatemic rickets. Conversely, augmenting cKL production might promote phosphate excretion in disorders of hypoparathyroidism or PTH resistance.

\section{Acknowledgments}

H. Jüppner is supported by grants R01DK46718 and P01DK011794 (subproject IV) from the National Institutes of Health. M. Wolf is supported by grants K24DK093723, R01DK081374, R01DK094796, and U54TR000255 from the National Institutes of Health.

Address correspondence to: Harald Jüppner, Massachusetts General Hospital Endocrine Unit, Thier 10, Boston, Massa- chusetts 02114, USA. Phone: 617.726.3966; Fax:617.726.7543; E-mail: hjueppner@ partners.org.

1. White KE, Bringhurst FR, Econs MJ. Genetic disorders of phosphate homeostasis. In: Jameson JL, Degroot LJ. Endocrinology. 6th ed. Philadelphia, Pennsylvania, USA; W.B. Saunders Company; 2010:1160-1175.

2. Gardella TJ, Jüppner H, Brown EM, Kronenberg HM, Potts JT Jr. Parathyroid hormone and parathyroid hormone-related peptide in the regulation of calcium homeostasis and bone development. In: Jameson JL, Degroot LJ. Endocrinology. 6th ed. Philadelphia, Pennsylvania, USA; W.B. Saunders Company; 2010:1040-1073.

3. Jüppner H, Wolf M, Salusky IB. FGF-23: More than a regulator of renal phosphate handling? J Bone Miner Res. 2010;25:2091-2097.

4. Urakawa I, et al. Klotho converts canonical FGF receptor into a specific receptor for FGF23. Nature. 2006;444(7120):770-774.

5. Kurosu H, et al. Regulation of fibroblast growth factor-23 signaling by Klotho. J Biol Chem. 2006;281(10):6120-6123.

6. Kuro-o M, et al. Mutation of the mouse klotho gene leads to a syndrome resembling ageing. Nature. 1997;390(6655):45-51.

7. Shimada T, et al. Targeted ablation of Fgf 23 demonstrates an essential physiological role of FGF23 in phosphate and vitamin D metabolism. J Clin Invest. 2004;113(4):561-568.

8. Sitara D, et al. Homozygous ablation of fibroblast growth factor-23 results in hyperphosphatemia and impaired skeletogenesis, and reverses hypophosphatemia in Phex-deficient mice. Matrix Biol. 2004;23(7):421-432.

9. Bonewald LF. The amazing osteocyte. J Bone Miner Res. 2011;26:229-238.

10. Perwad F, Azam N, Zhang M, Yamashita T, Tenenhouse H, Portale A. Dietary and serum phosphorus regulate fibroblast growth factor 23 expression and 1,25-dihydroxyvitamin D metabolism in mice. Endocrinology. 2005;146:5358-5364.

11. Burnett S, Gunawardene S, Bringhurst F, Jüppner $\mathrm{H}$, Lee H, Finkelstein J. Regulation of C-terminal and intact FGF-23 by dietary phosphate in men and women. J Bone Miner Res. 2006;21(8):1187-1196.

12. Smith RC, et al. Circulating $\alpha$ Klotho influences phosphate handling by controlling FGF23 production. J Clin Invest. 2012;122(12):4710-4715.

13. Wolf $M$. Update on fibroblast growth factor 23 in chronic kidney disease. Kidney Int. 2012; 82(7):737-747.

14. Brownstein CA, et al. A translocation causing increased alpha-klotho level results in hypophosphatemic rickets and hyperparathyroidism. Proc Natl Acad Sci U S A. 2008;105(9):3455-3460.

15. Ben-Dov IZ, et al. The parathyroid is a target organ for FGF23 in rats. J Clin Invest. 2007; 117(12):4003-4008.

16. Krajisnik T, et al. Fibroblast growth factor-23 regulates parathyroid hormone and 1alpha-hydroxylase expression in cultured bovine parathyroid cells. J Endocrinol. 2007;195:125-131.

17. Hu MC, et al. Klotho: a novel phosphaturic substance acting as an autocrine enzyme in the renal proximal tubule. FASAB J. 2010;24(9):3438-3450.

18. Segawa H, et al. Correlation between hyperphosphatemia and type II Na-Pi cotransporter activity in klotho mice. Am J Physiol Renal Physiol. 2007; 292(2):F769-F779.

19. Martin A, David V, Quarles LD. Regulation and function of the FGF23/klotho endocrine pathways. Physiol Rev. 2012;92(1):131-155.

20. Marsell R, et al. Gene expression analysis of kidneys from transgenic mice expressing fibroblast growth 
factor-23. Nephrol Dial Transplant. 2008;23:827-833. 21. Ichikawa $S$, et al. A homozygous missense mutation in human KLOTHO causes severe tumoral calcinosis. J Clin Invest. 2007;117(9):2684-2691.
22. Farrow EG, et al. Iron deficiency drives an autosomal dominant hypophosphatemic rickets (ADHR) phenotype in fibroblast growth factor-23 (Fgf23) knock-in mice. Proc Natl Acad Sci U S A. 2011;
108(46):E1146-E1155.

23. Farrow EG, Davis SI, Summers LJ, White KE. Initial FGF23-mediated signaling occurs in the distal convoluted tubule. J Am Soc Nephrol. 2009;20(5):955-960.

\title{
Understanding the TXA seizure connection
}

\author{
Debra A. Schwinn, ${ }^{1}$ G. Burkhard Mackensen, ${ }^{2}$ and Emery N. Brown ${ }^{3}$
}

\begin{abstract}
1Department of Anesthesiology, Pharmacology, and Biochemistry, Roy J. and Lucille A. Carver College of Medicine, The University of lowa, lowa City, lowa, USA. 2Department of Anesthesiology and Pain Medicine, University of Washington, Seattle, Washington, USA. ${ }^{3}$ Department of Brain and Cognitive Sciences, MIT-Harvard Division of Health Science and Technology, Massachusetts Institute of Technology, Cambridge, Massachusetts, USA.
\end{abstract}

\begin{abstract}
Transexamic acid (TXA) is an antifibrinolytic that has been used successfully to prevent blood loss during major surgery. However, as its usage has increased, there have been growing reports of postsurgical seizure events in cardiac surgery patients. In this issue of the JCI, Lecker et al. explore this connection and suggest that TXA-mediated inhibition of glycine receptors may underlie the effect. This finding prompted the authors to explore the preclinical efficacy of common anesthetics that function by reducing the TXA-mediated inhibition to prevent or modify postsurgical seizures.
\end{abstract}

\section{Introduction}

Translation from clinical bedside to laboratory bench and back is the holy grail of medicine. In this issue, Lecker et al. (1) undertake just such a translation. In an elegant set of studies, the authors investigate the neuronal mechanisms that underlie seizures occurring as side effects in patients receiving transexamic acid (TXA) to reduce blood loss during major cardiovascular surgery. Given the structural similarity between the antifibrinolytic drug TXA and inhibitory neurotransmitter-gated $\mathrm{Cl}^{-}$channel glycine receptors, the authors tested the hypothesis that TXA inhibits glycine receptor action as a possible mechanism underlying the seizures. After demonstrating that TXA inhibits glycine receptors, they then proposed that anesthetic agents with glycine receptor agonist properties may be uniquely suited to prevent such seizures. The studies presented are of high quality, based on an impressive range of laboratory data and models. However, they may be premature in recommending specific clinical actions to prevent seizures based on the limited clinical data. To place these interesting and provocative translational findings in context, we review use of antifibrinolytics in major cardiovascular surgery; this is followed by a brief overview of mechanisms of general anesthesia rec-

Conflict of interest: Debra A. Schwinn is a consultant for Watson Pharmaceuticals.

Citation for this article: J Clin Invest. 2012; 122(12):4339-4341. doi:10.1172/JCI66724 ognized to date and how anesthetic agents may play a role in treating seizures generally rather than specifically. We conclude by suggesting how the work of Lecker et al. moves the field forward, and where further work is still needed.

\section{Antifibrinolytic agents and major cardiovascular surgery}

Excessive perioperative bleeding remains a complication of cardiac surgery employing cardiopulmonary bypass, with blood transfusions considered a major contributor to subsequent morbidity and mortality (2). Despite serious efforts to achieve perioperative blood conservation by multimodal approaches (e.g., point-of-care monitoring paired with institution-specific blood transfusion algorithms and applied pharmacologic strategies), cardiac surgery patients continue to receive the vast majority of all perioperative blood transfusions (3). Therefore, antifibrinolytic therapy, because of its proven hemostatic effects, has become routine in this setting. Historically, three main antifibrinolytics have been used in cardiac surgery: lysine analogs $\varepsilon$-aminocaproic acid (EACA) and TXA in patients at low risk for bleeding, and the serine protease inhibitor aprotinin in patients at high risk for perioperative bleeding (such as those undergoing reoperations). However, with the removal of aprotinin from clinical practice following its association with a higher incidence of death and renal dysfunction in the Blood Conservation Using Anti-Fibrinolytics in a Randomized Trial (BART) study, many institutions worldwide have updated their therapeutic protocols $(4,5)$. The safety concerns surrounding aprotinin use that were raised in the BART study are largely backed by a recent systematic review and network meta-analysis confirming increased risk of mortality and renal morbidity with aprotinin versus TXA and EACA (6). The search for alternatives to aprotinin recently culminated in a phase 2 clinical trial demonstrating the inferiority of the novel protease inhibitor ecallantide in combating blood loss during high-risk cardiac surgery compared with TXA, and enhanced hemostatic efficacy with higher doses of TXA (7). However, as TXA usage has increased, there has been a growing awareness that some patients experience TXA-induced seizures. Occurrence peaks during the early postoperative period $(8)$ in a dose-related manner $(9,10)$, and seizures are more common in patients with a history of renal dysfunction (11).

\section{General anesthesia and seizures}

General anesthesia is critical for performing most surgical and many non-surgical interventions (12). This state is induced by administering an intravenous hypnotic such as propofol and maintained by a combination of inhaled drugs, hypnotics, opioids, and muscle relaxants. Understanding the mechanism whereby inhaled anesthetics create this drug-induced reversible coma has been a major area of investigation since the first use of ether in the 1840s. Substantial evidence has established that the molecular targets of inhaled anesthetic drugs in the brain and central nervous system are $\mathrm{GABA}_{\mathrm{A}}$, NMDA and glycine receptors, two-pore potassium channels, and HCN channels (13). Although immobility is now believed to result from inhaled anesthetics acting mostly at these targets in the spinal cord (14), the mechanisms of unconsciousness, amnesia, and analgesia 